\title{
Effects of whole body vibration training on body composition, skeletal muscle strength, and cardiovascular health
}

\author{
Song-Young Park ${ }^{1, *}$, Won-Mok Son², Oh-Sung Kwon ${ }^{3}$ \\ ${ }^{1}$ Whitaker Cardiovascular Institute, Boston University School of Medicine, Boston, MA, USA \\ ${ }^{2}$ Department of Physical Education, Pusan National University, Busan, Korea \\ ${ }^{3}$ Department of Internal Medicine, University of Utah, School of Medicine, Salt Lake City, UT, USA
}

Whole body vibration training (WBVT) has been used as a supplement to conventional exercise training such as resistance exercise training to improve skeletal muscle strength, specifically, in rehabilitation field. Recently, this exercise modality has been utilized by cardiovascular studies to examine whether WBVT can be a useful exercise modality to improve cardiovascular health. These studies reported that WBVT has not only beneficial effects on muscular strength but also cardiovascular health in elderly and disease population. However, its mechanism un- derlying the beneficial effects of WBVT in cardiovascular health has not been well documented. Therefore, this review highlighted the impacts of WBVT on cardiovascular health, and its mechanisms in conjunction with the improved muscular strength and body composition in various populations.

Keywords: Body composition, Vascular health, Whole body vibration

\section{INTRODUCTION}

The impact of impaired vascular function on cardiovascular disease (CVD) has been studied for several decades. In this regard, major factors for the development and progression of CVD are associated with attenuated arterial function including structural and functional changes in the arterial wall such as endothelial dysfunction, and arterial stiffness. In addition, many studies have reported that reduced muscular strength and reduced muscle mass with vascular aging and CVD (Figueroa et al., 2011a; Iemitsu et al., 2008; Otsuki et al., 2008). Exercise training has been known as nonpharmacological therapeutic modality to restore the impaired cardiovascular function.

Recently, whole body vibration (WBV), a novel training modality, has emerged as a useful exercise method for improving overall health (Fig. 1). In fact, previous studies have reported that WBV training (WBVT) alone improves body composition, mus- cular strength, and cardiovascular health concurrently. Also, WBVT is suitable for special populations such as the elderly and diseased populations who cannot perform traditional resistance or aerobic exercise training.

Accordingly, this review will highlight the impacts of WBVT on body composition such as muscle mass, fat mass, and skeletal muscle strength, and specifically, cardiovascular health. Furthermore, the mechanism underlying WBVT and the improvement in body composition and cardiovascular health will be also discussed.

\section{WHOLE BODY VIBRATION}

Conventional resistance exercise training uses additional mass to increase exercise intensities. However, the principles of WBV lie in the law of motion as stated by Sir Isaac Newton; mainly, that the force $(\mathrm{F})$ of an object is equal to the mass $(\mathrm{M})$ multiplied
${ }^{*}$ Corresponding author: Song-Young Park

Whitaker Cardiovascular Institute, Boston University School of Medicine, 840

Harrison Avenue Rm 520, Boston, MA 02118, USA

Tel: +1-617-638-5097, Fax: +1-801-582-1565, E-mail: songpark@bu.edu

Received: October 1, 2015 / Accepted: October 11, 2015
This is an Open Access article distributed under the terms of the Creative Commons Attribution Non-Commercial License (http://creativecommons.org/licenses/by-nc/3.0/) which permits unrestricted non-commercial use, distribution, and reproduction in any medium, provided the original work is properly cited. 


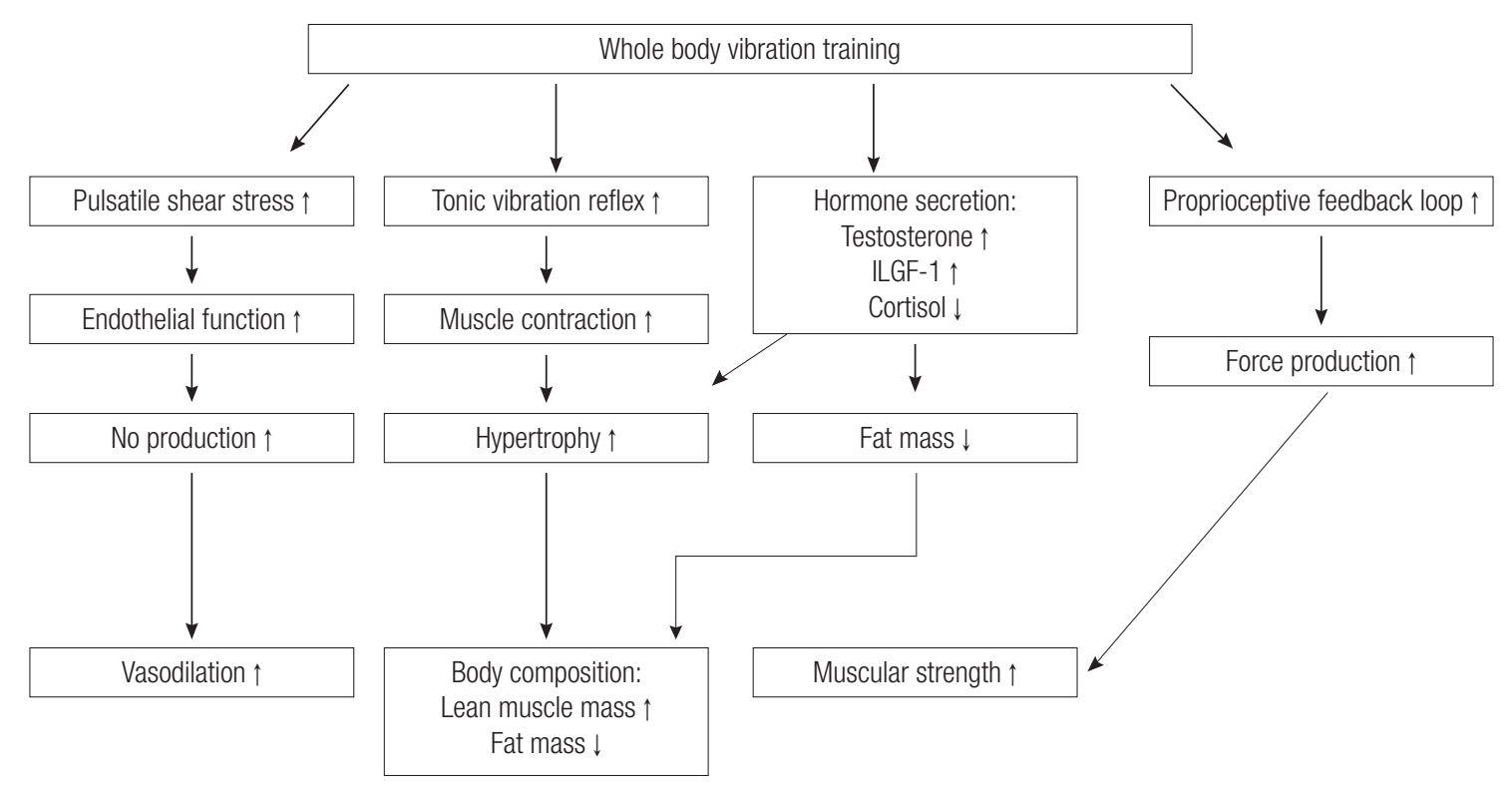

Fig. 1. Schematic diagram of the proposed mechanisms underlying the improved body composition and vascular function with whole body vibration training. ILGF-1, insulin-like growth factor 1.

by its acceleration (A) $(\mathrm{F}=\mathrm{M} \times \mathrm{A})$. Thus, functional force can be improved by either applying more mass or more acceleration to a body. WBV machines on the contrary, utilize acceleration by keeping the body weight constant (Cardinale and Bosco, 2003). This exercise mode uses an oscillating platform that delivers sinusoidal vibrations (Cochrane et al., 2008) that evoke reflexive muscle contractions (Bazrgari et al., 2008) while the person performs steadily controlled dynamic or static exercises. The mechanical action of vibration causes changes in the length of the muscle-tendon complex (Cardinale and Bosco, 2003) which in turn are detected by sensory receptors that modulate muscle contraction through reflex muscular activity via the stretch reflex loop and activation of the muscle spindles (Cardinale and Bosco, 2003). These contractions explain the increased levels muscle activity as reveled by electromyography in working muscles during WBV exercise (Cardinale and Lim, 2003; Hazell et al., 2008), and the increased energy output that is shown to result from the addition of vibration to squat exercises (Da Silva et al., 2007). The effect of vibration is dependent upon the amplitude and frequency of oscillations, and therefore the rate and length of muscular stretch. The amplitude is determined by the peak to peak displacement of the platform, with the repetition rate of cycles of oscillations denoted by the frequency of the vibration (measured in $\mathrm{Hz}$ ) (Cardinale and Bosco, 2003). The vibration effects on leg muscle activity and have been shown to vary with different frequencies of vibration. Greater vibration frequencies $(35-45 \mathrm{~Hz})$ have been shown to in- crease greater muscle activity than lower frequencies $(<35 \mathrm{~Hz})$ for both static and dynamic exercises (Hazell et al., 2007).

There are different types of vibration platforms commonly used in clinical studies including oscillating side to side action of the platforms, uniformly up and down action and triplane in which the vibration is applied in anterior/posterior, side to side, and up and down directions

\section{WBVT AND MUSCLE MASS}

Research on the effects WBVT on body composition is more extensive than in the cardiovascular system. Several studies have shown increases in muscle mass in the young (Figueroa et al., 2012; Milanese et al., 2012; Roelants et al., 2004) as well as in clinical populations (Bogaerts et al., 2007; Machado et al., 2010). Following 24 weeks of WBVT, which utilized static and dynamic leg and arm exercises $3 \times$ week, Roelants et al. (2004) showed increases in total muscle mass in a group of 13 young females. A recent project by our group in young overweight/obese women (Figueroa, et al., 2014a) demonstrated that six weeks of WBVT using four leg exercises resulted in improvements in leg lean mass, assessed by dual-energy X-ray absorptiometry (DXA), in comparison to baseline. Although the changes in the WBV group over time were not significantly different compared to the control group, these changes are clinically relevant since the increase in leg muscle mass of $\sim 0.4 \mathrm{~kg}(0.9 \mathrm{lb})$ seems to be important consid- 
ering the short amount of time of the intervention. In a study by Milanese et al. (2012), WBVT completed over an 8-week intervention period resulted in increased total body lean mass in young nonobese women. In another 10-week study, Machado et al. (2010) found a significant increase in thigh muscles cross sectional area in healthy elderly women. The increased in cross sectional area (assessed by computed tomography) was a result of increases in the vastus medialis and bicep femoris by $8.7 \%$ and $15.5 \%$, respectively. Based on the previous results, we conducted a study of 12 weeks of WBVT in postmenopausal 23 women with a similar protocol as the one used by Machado et al. (2010). Although we saw no significant changes in leg lean mass (assessed by DXA), there was a trend for it to increase after increase after WBVT $(\sim 0.3 \mathrm{~kg}, P=0.06)$. The impact of WBVT on muscle mass over a period longer than 6 months has only been studied once. In this study by Bogaerts et al. (2007), elderly men trained 3 times weekly over a period of one year. These individuals were split into one of two groups that performed a WBVT or a fitness training (aerobic, resistance, balance, and flexibility exercises) routine. At the end of this investigation, leg muscle mass was significantly increased $3.4 \%$ in the WBVT group, but not different in the fitness (3.8\%) groups. It was therefore concluded that WBVT is as efficient as a fitness intervention in increasing muscle mass in elderly men.

\section{WBVT AND FAT MASS}

Previous animal research in rats suggests that chronic exposure to WBV decreases adipogenesis (Maddalozzo et al., 2008; Rubin et al., 2007). Vissers et al. (2010) found that WBVT would have a greater potential to reduce visceral adipose tissue than a program consisting of combined aerobic and resistance training (RT) in middle-aged obese adults. It has been previously reported that a combination of RT and WBVT, but not RT alone, was effective for decreasing total body fat percentage after 8 months of training in postmenopausal women (Fjeldstad et al., 2009) and after eight weeks of training in young adults (Artero et al., 2012). Alternatively, Verschueren et al. (2004) suggested that 6 months of RT and WBVT produce similar decreases $(-3.1 \%$ and $-2.3 \%)$ in fat mass measured by DXA in older women. Furthermore, Lamont et al. (2011) reported decreases in leg fat\% after six weeks of combined RT and WBVT using squat exercises, an effect that was not achieved by RT alone. In a recent study by Milanese et al. (2012), it was found that eight weeks of WBVT decreased leg and total body fat mass in young nonobese women. However, a recent study by our group showed no changes in total fat mass in overweight/ obese premenopausal women after six of WBVT (Figueroa et al., 2012). In another study by our group, 12 weeks of WBVT did not change total body fat percentage in obese postmenopausal women (Figueroa et al., 2014b). Although our results failed to show decreases in total fat mass and percentage, we did not measure leg fat mass or percentage, which has been previously shown to improve after eight weeks of WBVT (Milanese et al., 2012) or WBVT combined with RT (Artero et al., 2012) in young adults. Nevertheless, based on these previous results, it is feasible to propose that decreases in leg fat mass and fat percentage after WBVT are attainable.

\section{WBVT AND STRENGTH}

Recent evidence suggests that WBV exercise could be an alternative exercise modality for eliciting muscle strength in older adults (Bogaerts et al., 2007; Kawanabe et al., 2007; Machado et al., 2010; Tapp and Signorile, 2014). Findings of improved strength have also been shown following WBVT in younger individuals (Figueroa et al., 2010; Figueroa et al., 2012; Roelants et al., 2004). The addition of vibration to exercise programs has been shown to increase strength and power more than exercise programs without vibration (Kawanabe et al., 2007; Rønnestad, 2004). Moreover, recent studies have shown that WBVT using a frequency of $25-40 \mathrm{~Hz}$ and an amplitude of $1-2 \mathrm{~mm}$ for 6 months can increase muscle strength at a similar extent than a RT program in postmenopausal women (Schwedhelm et al., 2008; Verschueren et al., 2004). In a recent study that investigated the effects of 1-year WBVT in elderly men, this modality proved to be as effective as a fitness program (combination of aerobic, resistance, and flexibility exercises) for increasing knee extension strength (Bogaerts et al., 2007). According to Roelants et al. (2004) a significant $24.4 \%$ net benefit increase in isometric knee extensor strength was observed after 6 months of WBVT in young untrained females. The results of this previous study in young women were corroborated by another study by our group, which showed improvements in leg extension strength after a 6week WBVT program in young overweight and obese women (Figueroa et al., 2012). Additionally, increases in isometric (15\%) and dynamic strength (16\%) of the knee extensor muscles have been shown after 6 months (Roelants et al., 2004; Verschueren et al., 2004) of WBVT in postmenopausal women. Shorter duration interventions have also demonstrated that WBVT is a feasible intervention to increase muscle strength in postmenopausal women. 
Following 10 weeks of WBVT using leg exercises with a frequency of $3 \times$ week, Machado et al. (2010) demonstrated increases (38.8\%) in maximal voluntary isometric contraction of the leg extensors. In a study by our group, leg strength increased after 6 (9\%) (Figueroa et al., 2012) and 12 weeks (21\%) (Figueroa et al., 2014b) of WBVT in postmenopausal women.

\section{WBVT FOR VASCULAR HEALTH}

The beneficial effects of WBV on the cardiovascular system have been mostly observed following acute bouts of WBV. These changes include local increases in skin blood flow in the upper (Maloney-Hinds et al., 2008) and lower extremities (Lohman 3rd et al., 2007) after a session of passive vibration (PV), defined as 21 exposure of the limbs to continuous vibration without performing voluntary muscle contractions. The vasodilatory response in the previous studies was found to be related to increases in nitric oxide (NO) production (Maloney-Hinds et al., 2009). In addition to $\mathrm{NO}$, other endothelial factors such as reduced endothelin-1, a vasoconstrictor, may be implicated in the vasodilatory response to PV (Nakamura et al., 1996). Another line of studies by our group concluded that augmentation index (Aix) (Sanchez-Gonzalez et al., 2012), leg pulse wave velocity (PWV) and brachial and ankle pulse wave velocity (baPWV) (Wong et al., 2012) decrease while aortic PWV did not change (Wong et al., 2012) after a 10-min session of PV on the legs in healthy young men. Similar results to PV have also been seen after a WBV session. Otsuki et al. (2008) found decreases in baPWV after a session of ten 1-min static squat exercises with WBV separated by 1-min interset rest periods; In addition, a study by our group showed a decrease in AIx and legPWV (Figueroa et al., 2011b) following the same protocol used by Otsuki et al. (2008). Increases in blood flow after an acute session of WBV were demonstrated by Kerschan-Schindl et al. (2001) who found increased blood flow in the popliteal artery after 9 minutes of static squat with WBV. Increases in blood flow were also demonstrated by Yamada et al. (2005) who found that blood volume in the vastus lateralis acutely increases after WBV with a dynamic squat exercise. Research on the chronic effects of WBVT on the cardiovascular system is limited to studies by our group. Recently, we reported promising findings that may lead to the application of WBVT as a method of cardiovascular therapy. In this investigation, 10 overweight/obese normotensive women underwent 6 weeks of WBVT using leg exercises. WBVT resulted in a decrease in brachial systolic BP (SBP), aortic SBP, baPWV, Aix, and sympathovagal balance (Figueroa et al., 2012). In anoth- er line of studies by our group in obese postmenopausal women, similar decreases were reported in aortic SBP, aortic DBP, and AIx after 6 weeks of WBVT (Figueroa et al., 2014a), and brachial SBP and DBP and baPWV after 12 weeks of WBVT (Figueroa et al., 2012). The decreases in baPWV in postmenopausal women were attributed to decreases in legPWV, since there were no changes in aortic PWV (Figueroa et al., 2012). Hence, the effect of WBVT on arterial stiffness is localized to the trained legs. Furthermore, Sañudo et al. (2013) reported increases in femoral artery blood flow and mean velocity suggesting improvements in leg artery vasodilation after 12 weeks of WBVT in individuals with type 2 diabetes mellitus. However, our previous studies have not investigated the mechanisms behind the improvements in arterial function after WBVT, which represents a gap in knowledge. Since the elderly have increased SBP, wave reflection and arterial stiffening the likelihood of cardiovascular events may be increased in these individuals. This is important to point out since the chronic adaptations to WBVT reported in the previously mentioned studies could translate into health benefits for the elderly.

\section{PROPOSED MECHANISMS OF WBVT FOR IMPROVED STRENGTH, BODY COMPOSITION, AND CARDIOVASCULAR HEALTH}

There have been a number of mechanisms proposed for the increases in strength associated with WBV including the tonic vibration reflex (Cardinale and Bosco, 2003), increased hormone secretion (Bosco et al., 1999; Bosco et al., 2000; Cardinale et al., 2010), muscle hypertrophy (Delecluse et al., 2003) and stimulation of proprioceptive pathways (Delecluse et al., 2003). Tonic vibration reflex happens when vibration causes a reflex muscle contraction by exciting the muscle spindles in the exposed muscle (Cardinale and Bosco, 2003). Previous studies in rats have shown enlargement of fast and slow twitch muscle fibers following shortterm WBV exposure (Necking et al., 1996). In addition, several human studies have shown a vibration induced muscle hypertrophy in healthy individuals as well as in clinical populations (Bogaerts et al., 2007; Figueroa et al., 2014b; Machado et al., 2010; Milanese et al., 2012; Roelants et al., 2004), which cannot be overlooked as possibility for strength improvement. It is possible that WBVT may improve the efficiency of the proprioceptive feedback loop. Proprioceptive pathways are used during isometric contractions to produce force (Gandevia, 2001). A more efficient proprioceptive feedback loop, caused by WBV, could lead to in- 
creases in force production. There are some mechanisms proposed for the improvement in body composition (increase in muscle mass and decrease fat mass) with WBVT, which are the same mechanisms that cause improvements following RT. Previous studies in rats have shown enlargement of types I and II muscle fibers following vibration (Necking et al., 1996). Interestingly, it was previously reported that the additional gravitational load that an individual experiences when exposed to WBV elicits an anabolic hormonal response (Bosco et al., 1999). This would be comparable to mechanistic studies using conventional RT, which found a positive relationship between increasing blood levels of anabolic hormones and increased muscle mass and strength (Ahtiainen et al., 2003; Kraemer and Ratamess, 2005). In fact, an acute session of WBV has been shown to acutely increase serum levels of testosterone and human growth hormone and decreases of cortisol levels (Bosco et al., 2000). In addition, previous animal research in rats suggests that chronic exposure to WBV stimulates a lipolytic effect (Maddalozzo et al., 2008). Rittweger et al. (2002) reported that metabolic power increased with vibration training and that energy requirements during vibration training were similar to those of moderate intensity walking. Indeed, an acute bout of WBV transiently reduces plasma glucose, possibly by increasing glucose uptake and utilization by contracting muscle (Di Loreto et al., 2004). These findings suggest that WBVT has potential as a treatment for obesity and sarcopenia by inducing a predominance of anabolic hormones along with increased energy expenditure that may lead to an increase in lean mass and reduction in fat mass. This is very important to mention since the progression of arterial stiffness in older individuals is related to muscle tissue loss (Rolland et al., 2008). It is possible that the same mechanisms that explain improved vasodilation following aerobic training and RT are those causing vascular improvements with WBVT. It has been speculated that when the body is exposed to vibration it evokes rhythmic muscle contractions (Da Silva et al., 2007; Kerschan-Schindl et al., 2001) which may induce changes in peripheral arteries. Widening of the capillaries in the gastrocnemius and quadriceps facilitates the exchange of nutrients, metabolic byproducts and delivery of oxygen between the capillaries and the fluid surrounding body cells (Kerschan-Schindl et al., 2001). WBV has been shown to increase mean velocity of the popliteal artery, which indicates vasodilation of the small vessels in the exposed muscles. This increase in blood flow may be due to vibration-induced reduction in blood viscosity (Kerschan-Schindl et al., 2001). Another factor that may play a role in the increased peripheral blood flow might be an improved endothelial function.
The underlying mechanism for the significant increase in blood flow following vibration may be due to pulsatile endothelial stress resulting in increased endothelial nitric oxide synthase activity and NO concentration (Maloney-Hinds et al., 2009; Rubin et al., 2007). In addition to NO, other endothelial factors such as reduced endothelin-1, a vasoconstrictor, may be implicated in the vasodilatory response to vibration (Nakamura et al., 1996).

\section{CONCULSIONS}

In summary, the WBVT has been shown to improve body composition, muscular strength, and cardiovascular health. Especially, WBVT is useful exercise modality for disease and elderly population because the WBVT does not require utilizing conventional dynamic exercise such as free weight or dynamic movement. Instead of using those conventional exercise modalities, the WBVT is utilizing vibration, oscillatory shear stress, which evokes not only muscular contraction but also local vasodilation in the microcirculation by the stimulation of endothelium. WBVT is an efficient exercise modality for improving both skeletal muscle strength and cardiovascular health, specifically for elderly and disease populations. Also, this exercise modality, WBVT, can be further utilized for immobile population to improve skeletal muscle strength and cardiovascular health simultaneously.

\section{CONFLICT OF INTEREST}

No potential conflict of interest relevant to this article was reported.

\section{REFERENCES}

Ahtiainen JP, Pakarinen A, Alen M, Kraemer WJ, Häkkinen K. Muscle hypertrophy, hormonal adaptations and strength development during strength training in strength-trained and untrained men. Eur J Appl Physiol 2003;89:555-563.

Artero EG, Espada-Fuentes JC, Argüelles-Cienfuegos J, Román A, Gómez-López PJ, Gutiérrez A. Effects of whole-body vibration and resistance training on knee extensors muscular performance. Eur J Appl Physiol 2012;112:1371-1378.

Bazrgari B, Shirazi-Adl A, Kasra M. Seated whole body vibrations with high-magnitude accelerations--relative roles of inertia and muscle forces. J Biomech 2008;41:2639-2646.

Bogaerts A, Delecluse C, Claessens AL, Coudyzer W, Boonen S, Ver- 
schueren SM. Impact of whole-body vibration training versus fitness training on muscle strength and muscle mass in older men: a 1-year randomized controlled trial. J Gerontol A Biol Sci Med Sci 2007;62:630635.

Bosco C, Colli R, Introini E, Cardinale M, Tsarpela O, Madella A, Tihanyi J, Viru A. Adaptive responses of human skeletal muscle to vibration exposure. Clin Physiol 1999;19:183-187.

Bosco C, Iacovelli M, Tsarpela O, Cardinale M, Bonifazi M, Tihanyi J, Viru M, De Lorenzo A, Viru A. Hormonal responses to whole-body vibration in men. Eur J Appl Physiol 2000;81:449-454.

Cardinale M, Bosco C. The use of vibration as an exercise intervention. Exerc Sport Sci Rev 2003;31:3-7.

Cardinale M, Lim J. Electromyography activity of vastus lateralis muscle during whole-body vibrations of different frequencies. J Strength Cond Res 2003;17:621-624.

Cardinale M, Soiza RL, Leiper JB, Gibson A, Primrose WR. Hormonal responses to a single session of wholebody vibration exercise in older individuals. Br J Sports Med 2010;44:284-288.

Cochrane DJ, Sartor F, Winwood K, Stannard SR, Narici MV, Rittweger J. A comparison of the physiologic effects of acute whole-body vibration exercise in young and older people. Arch Phys Med Rehabil 2008;89:815821.

Da Silva ME, Fernandez JM, Castillo E, Nuñez VM, Vaamonde DM, Poblador MS, Lancho JL. Influence of vibration training on energy expenditure in active men. J Strength Cond Res 2007;21:470-475.

Delecluse C, Roelants M, Verschueren S. Strength increase after wholebody vibration compared with resistance training. Med Sci Sports Exerc 2003;35:1033-1041.

Di Loreto C, Ranchelli A, Lucidi P, Murdolo G, Parlanti N, De Cicco A, Tsarpela O, Annino G, Bosco C, Santeusanio F, Bolli GB, De Feo P. Effects of whole-body vibration exercise on the endocrine system of healthy men. J Endocrinol Invest 2004;27:323-327.

Figueroa A, Gil R, Wong A, Hooshmand S, Park SY, Vicil F, Sanchez-Gonzalez MA. Whole-body vibration training reduces arterial stiffness, blood pressure and sympathovagal balance in young overweight/ obese women. Hypertens Res 2012;35:667-672.

Figueroa A, Hooshmand S, Figueroa M, Bada AM. Cardiovagal baroreflex and aortic hemodynamic responses to isometric exercise and post-exercise muscle ischemia in resistance trained men. Scand J Med Sci Sports 2010;20:305-309.

Figueroa A, Kalfon R, Madzima TA, Wong A. Effects of whole-body vibration exercise training on aortic wave reflection and muscle strength in postmenopausal women with prehypertension and hypertension. J Hum Hypertens 2014a;28:118-122.

Figueroa A, Kalfon R, Madzima TA, Wong A. Whole-body vibration ex- ercise training reduces arterial stiffness in postmenopausal women with prehypertension and hypertension. Menopause 2014b;21:131136.

Figueroa A, Park SY, Seo DY, Sanchez-Gonzalez MA, Baek YH. Combined resistance and endurance exercise training improves arterial stiffness, blood pressure, and muscle strength in postmenopausal women. Menopause 2011a;18:980-984.

Figueroa A, Vicil F, Sanchez-Gonzalez MA. Acute exercise with wholebody vibration decreases wave reflection and leg arterial stiffness. Am J Cardiovasc Dis 2011b;1:60-67.

Fjeldstad C, Palmer IJ, Bemben MG, Bemben DA. Whole-body vibration augments resistance training effects on body composition in postmenopausal women. Maturitas 2009;63:79-83.

Gandevia SC. Spinal and supraspinal factors in human muscle fatigue. Physiol Rev 2001;81:1725-1789.

Hazell TJ, Jakobi JM, Kenno KA. The effects of whole-body vibration on upper- and lower-body EMG during static and dynamic contractions. Appl Physiol Nutr Metab 2007;32:1156-1163.

Hazell TJ, Thomas GW, Deguire JR, Lemon PW. Vertical whole-body vibration does not increase cardiovascular stress to static semi-squat exercise. Eur J Appl Physiol 2008;104:903-908.

Iemitsu M, Maeda S, Otsuki T, Sugawara J, Kuno S, Ajisaka R, Matsuda M. Arterial stiffness, physical activity, and atrial natriuretic Peptide gene polymorphism in older subjects. Hypertens Res 2008;31:767-774.

Kawanabe K, Kawashima A, Sashimoto I, Takeda T, Sato Y, Iwamoto J. Effect of whole-body vibration exercise and muscle strengthening, balance, and walking exercises on walking ability in the elderly. Keio J Med 2007;56:28-33.

Kerschan-Schindl K, Grampp S, Henk C, Resch H, Preisinger E, Fialka-Moser V, Imhof H. Whole-body vibration exercise leads to alterations in muscle blood volume. Clin Physiol 2001;21:377-382.

Kraemer WJ, Ratamess NA. Hormonal responses and adaptations to resistance exercise and training. Sports Med 2005;35:339-361.

Lamont HS, Cramer JT, Bemben DA, Shehab RL, Anderson MA, Bemben MG. Effects of a 6-week periodized squat training with or without whole-body vibration upon short-term adaptations in squat strength and body composition. J Strength Cond Res 2011;25:1839-1848.

Lohman EB 3rd, Petrofsky JS, Maloney-Hinds C, Betts-Schwab H, Thorpe D. The effect of whole body vibration on lower extremity skin blood flow in normal subjects. Med Sci Monit 2007;13:CR71-76.

Machado A, García-López D, González-Gallego J, Garatachea N. Wholebody vibration training increases muscle strength and mass in older women: a randomized-controlled trial. Scand J Med Sci Sports 2010;20:200-207.

Maddalozzo GF, Iwaniec UT, Turner RT, Rosen CJ, Widrick JJ. Whole- 
body vibration slows the acquisition of fat in mature female rats. Int J Obes (Lond) 2008;32:1348-1354.

Maloney-Hinds C, Petrofsky JS, Zimmerman G. The effect of $30 \mathrm{~Hz}$ vs. 50 $\mathrm{Hz}$ passive vibration and duration of vibration on skin blood flow in the arm. Med Sci Monit 2008;14:CR112-116.

Maloney-Hinds C, Petrofsky JS, Zimmerman G, Hessinger DA. The role of nitric oxide in skin blood flow increases due to vibration in healthy adults and adults with type 2 diabetes. Diabetes Technol Ther 2009;11: 39-43.

Milanese C, Piscitelli F, Simoni C, Pugliarello R, Zancanaro C. Effects of whole-body vibration with or without localized radiofrequency on anthropometry, body composition, and motor performance in young nonobese women. J Altern Complement Med 2012;18:69-75.

Nakamura H, Okazawa T, Nagase H, Yoshida M, Ariizumi M, Okada A. Change in digital blood flow with simultaneous reduction in plasma endothelin induced by hand-arm vibration. Int Arch Occup Environ Health 1996;68:115-119.

Necking LE, Lundström R, Lundborg G, Thornell LE, Fridén J. Skeletal muscle changes after short term vibration. Scand J Plast Reconstr Surg Hand Surg 1996;30:99-103.

Otsuki T, Takanami Y, Aoi W, Kawai Y, Ichikawa H, Yoshikawa T. Arterial stiffness acutely decreases after whole-body vibration in humans. Acta Physiol (Oxf) 2008;194:189-194.

Rittweger J, Ehrig J, Just K, Mutschelknauss M, Kirsch KA, Felsenberg D. Oxygen uptake in whole-body vibration exercise: influence of vibration frequency, amplitude, and external load. Int J Sports Med 2002;23: 428-432.

Roelants M, Delecluse C, Goris M, Verschueren S. Effects of 24 weeks of whole body vibration training on body composition and muscle strength in untrained females. Int J Sports Med 2004;25:1-5.

Rolland Y, Czerwinski S, Abellan Van Kan G, Morley JE, Cesari M, Onder G, Woo J, Baumgartner R, Pillard F, Boirie Y, Chumlea WM, Vellas B. Sarcopenia: its assessment, etiology, pathogenesis, consequences and future perspectives. J Nutr Health Aging 2008;12:433-450.

Rønnestad BR. Comparing the performance-enhancing effects of squats on a vibration platform with conventional squats in recreationally resistance-trained men. J Strength Cond Res 2004;18:839-845.

Rubin CT, Capilla E, Luu YK, Busa B, Crawford H, Nolan DJ, Mittal V, Rosen CJ, Pessin JE, Judex S. Adipogenesis is inhibited by brief, daily exposure to high-frequency, extremely low-magnitude mechanical signals. Proc Natl Acad Sci U S A 2007;104:17879-17884.

Sanchez-Gonzalez MA, Wong A, Vicil F, Gil R, Park SY, Figueroa A. Impact of passive vibration on pressure pulse wave characteristics. J Hum Hypertens 2012;26:610-615.

Sañudo B, Alfonso-Rosa R, Del Pozo-Cruz B, Del Pozo-Cruz J, Galiano D, Figueroa A. Whole body vibration training improves leg blood flow and adiposity in patients with type 2 diabetes mellitus. Eur J Appl Physiol 2013;113:2245-2252.

Schwedhelm E, Maas R, Freese R, Jung D, Lukacs Z, Jambrecina A, Spickler W, Schulze F, Böger RH. Pharmacokinetic and pharmacodynamic properties of oral L-citrulline and L-arginine: impact on nitric oxide metabolism. Br J Clin Pharmacol 2008;65:51-59.

Tapp LR, Signorile JF. Efficacy of WBV as a modality for inducing changes in body composition, aerobic fitness, and muscular strength: a pilot study. Clin Interv Aging 2014;9:63-72.

Verschueren SM, Roelants M, Delecluse C, Swinnen S, Vanderschueren D, Boonen S. Effect of 6-month whole body vibration training on hip density, muscle strength, and postural control in postmenopausal women: a randomized controlled pilot study. J Bone Miner Res 2004; 19:352-359.

Vissers D, Verrijken A, Mertens I, Van Gils C, Van de Sompel A, Truijen S, Van Gaal L. Effect of long-term whole body vibration training on visceral adipose tissue: a preliminary report. Obes Facts 2010;3:93-100.

Wong A, Sanchez-Gonzalez MA, Gil R, Vicil F, Park SY, Figueroa A. Passive vibration on the legs reduces peripheral and systemic arterial stiffness. Hypertens Res 2012;35:126-127.

Yamada E, Kusaka T, Miyamoto K, Tanaka S, Morita S, Tanaka S, Tsuji S, Mori S, Norimatsu H, Itoh S. Vastus lateralis oxygenation and blood volume measured by near-infrared spectroscopy during whole body vibration. Clin Physiol Funct Imaging 2005;25:203-208. 